\title{
De la modélisation de l'activité humaine à la modélisation pour la simulation sociale : entre réalisme et fécondité technologique
}

From modeling human activity to modeling for social simulation:

between realism and technological innovation

Yvon Haradji, Julien Guibourdenche, Quentin Reynaud, Germain Poizat, Nicolas Sabouret, François Sempé, Thomas Huraux et Mariane Galbat

\section{(2) OpenEdition}

Journals

Édition électronique

URL : http://journals.openedition.org/activites/3106

DOI : 10.4000/activites.3106

ISSN : 1765-2723

Éditeur

ARPACT - Association Recherches et Pratiques sur les ACTivités

Référence électronique

Yvon Haradji, Julien Guibourdenche, Quentin Reynaud, Germain Poizat, Nicolas Sabouret, François Sempé, Thomas Huraux et Mariane Galbat, « De la modélisation de l'activité humaine à la modélisation pour la simulation sociale : entre réalisme et fécondité technologique », Activités [En ligne], 15-1 | 2018, mis en ligne le 15 avril 2018, consulté le 19 avril 2019. URL : http:// journals.openedition.org/activites/3106; DOI : 10.4000/activites.3106

Ce document a été généré automatiquement le 19 avril 2019

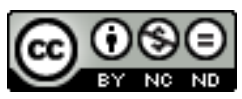

Activités est mis à disposition selon les termes de la licence Creative Commons Attribution - Pas d'Utilisation Commerciale - Pas de Modification 4.0 International. 


\title{
De la modélisation de l'activité
} humaine à la modélisation pour la simulation sociale : entre réalisme et fécondité technologique

\author{
From modeling human activity to modeling for social simulation: \\ between realism and technological innovation
}

Yvon Haradji, Julien Guibourdenche, Quentin Reynaud, Germain Poizat, Nicolas Sabouret, François Sempé, Thomas Huraux et Mariane Galbat

\section{NOTE DE L'ÉDITEUR}

Article soumis le 14/11/2017, accepté le 25/02/2018

\section{Introduction}

1 Nous sommes engagés depuis quelques années dans la conception d'une plateforme de simulation sociale'. SMACH (Simulation Multi-Agent de l'ACtivité Humaine) s'inscrit pour EDF dans un cadre de recherche et développement visant à développer ce type de simulation pour l'anticipation et la réduction des consommations d'énergie dans l'habitat. C'est pour répondre à ce défi de maîtrise de l'efficacité énergétique que nous souhaitons pouvoir simuler l'impact d'un tarif sur la consommation individuelle ou collective, tester les conséquences d'un nouvel équipement sur la facture du client, évaluer l'effet d'une politique énergétique à l'échelle locale, régionale ou nationale.

Dans cet article, nous proposons d'articuler structurellement la simulation avec l'activité humaine car la consommation dans l'habitat résulte pour beaucoup de la dynamique de vie quotidienne (préparer le repas, donner le bain aux enfants, laver le linge, etc.) en 
interaction avec les appareils et l'environnement. Pour cela, nous développons une simulation sociale qui cherche à reproduire des interactions humaines (Manzo, 2007; Phan, \& Amblard, 2007 ; Phan, \& Varenne, 2010 ; Sanders, 2007) dans l'habitat pour créer un réalisme dans la dynamique des consommations énergétiques. La plateforme SMACH est aujourd'hui beaucoup sollicitée, principalement pour sa capacité à produire des courbes de consommation réalistes (cf. Figure 2). Son efficacité calculatoire dépend pour beaucoup du réalisme de l'activité humaine simulée. Ainsi, cet article se focalise sur la relation structurante de la simulation à l'activité humaine. Il aborde la question du niveau de réalisme nécessaire pour produire des modèles artificiels suffisamment féconds sur le plan technologique.

Pour cela, nous présentons en section 2 les systèmes multi-agents (SMA) comme une solution intéressante pour approcher la complexité de l'activité humaine, notamment dans le cadre de projet d'efficience énergétique. Dans cette section nous présentons également le principe de fonctionnement de la plateforme SMACH. Dans la section 3 nous présentons l'énaction comme un cadre théorique pertinent pour penser l'articulation entre une épistémologie du vivant et de l'artificiel. Les sections 4, 5, et 6 montrent des proximités et des différences entre la connaissance empirique de l'activité et la simulation de l'activité humaine. Dans la dernière section nous confrontons la simulation à une situation réelle. Nous proposons ainsi avec cet article une réflexion sur la conception de modèles pour la simulation et sur leur capacité à simuler une activité humaine réaliste dans l'habitat.

\section{Simuler l'activité humaine dans l'habitat pour des calculs d'efficience énergétique}

\subsection{La Simulation Multi-Agent : un moyen pour approcher la complexité de l'activité humaine dans l'habitat}

La simulation est un outil méthodologique connu en ergonomie. Elle a notamment pour fonction d'anticiper une situation future et d'aider à la décision des concepteurs (Béguin, \& Weill-Fassina, 1997). L'environnement de travail est simulé (espace de travail transformé, maquette d'architecture ou d'interface, simulateur échelle 1, etc.) et l'activité des opérateurs y est mise en scène pour la simulation organisationnelle (e.g., Van Belleghem, \& Guerry, 2016). Un environnement simulé est très intéressant pour l'exploration de certains phénomènes comportementaux et expérientiels difficiles à appréhender en situation réelle (Gallagher, Reinerman-Jones, Sollins, \& Janz, 2014).

Depuis quelques années, les simulations de type multi-agent ont fait leur apparition en ergonomie. L'objectif des Systèmes Multi-Agents (Ferber, 1995) consiste en : 1) une analyse des mécanismes d'auto-organisation des systèmes complexes (vivants, sociaux, artificiels) émergeant au cours de l'interaction entre plusieurs entités autonomes (e.g. les agents d'une société); 2) la conception de systèmes artificiels dotés de capacités d'interaction et de coopération pour accomplir une tâche. En ergonomie, des simulations de ce type ont principalement été utilisées dans l'optique de concevoir des systèmes coopératifs complexes (e.g., Dugdale, Pavard, \& Soubie, 2000). Les chercheurs sont partis d'un constat simple: la coopération en situations de travail est la plupart du temps imprévisible du fait de son caractère indéterminé, distribué et contextualisé. Ils se sont 
alors appuyés sur les apports conceptuels et méthodologiques des approches de la complexité pour comprendre et modéliser avec les systèmes multi-agents (SMA) l'autoorganisation dans les systèmes sociotechniques coopératifs (Pavard, \& Dugdale, 2006). L'idée première est qu'une interaction locale entre les agents et leur environnement peut générer des comportements émergents dynamiques et complexes. Le développement de simulations multi-agents a permis d'orienter des décisions concernant le fonctionnement collectif global en centre d'appel du SAMU, dans le contrôle aérien, ou lors de la mise en œuvre d'un plan d'urgence (Bellamine-Ben Saoud, \& al., 2006 ; Dugdale, \& al., 2000).

6 Les simulations multi-agents sont des outils méthodologiques intéressants pour la conception: compréhension des phénomènes, évaluation des solutions, recherche d'alternatives, concertation, validation, aide à la décision. Les simulations multi-agents sont prédictives mais dans un sens beaucoup plus faible que celui utilisé classiquement (Haradji, \& Poizat, 2017). Il ne s'agit pas de moyens d'administration de la preuve. Le principe consiste à jouer sur un ensemble de variables exogènes de l'environnement pour explorer les transformations de la situation (l'introduction d'un nouveau type de chauffage) et évaluer leurs impacts sur des variables endogènes jugées pertinentes (l'impact de ce chauffage sur le confort ressenti par les agents en fonction de leur activité). La simulation fonctionne ainsi comme un «laboratoire virtuel» complémentaire à l'analyse de l'activité. C'est un moyen pour "ouvrir l'espace de conception » et nourrir l'interaction (discussion et négociation) entre les acteurs engagés dans la conception autour de différents choix possibles (Salembier, \& Pavard, 2004), en complément de l'analyse d'activité.

\subsection{SMACH : le réalisme de l'activité humaine pour des problématiques d'efficience énergétique}

7 Dans le cadre des problématiques sur l'efficience énergétique, les simulations sont nécessaires car nous disposons de peu de connaissances nous permettant de comprendre et d'agir sur les niveaux ou pics de consommation comme l'indique Grandjean (2013). Les travaux actuels à partir d'expérimentation en situation réelle ou de panels portent très souvent sur l'enregistrement des consommations. Ils ne permettent pas de comprendre la relation entre comportements humains dans l'habitat et consommation. De plus, ces approches ne disposent pas de la souplesse nécessaire pour tester des hypothèses et en voir l'impact sur la courbe de consommation locale ou globale. La simulation permet de dépasser ces limites mais Plessis, Amouroux et Haradji (2014) ou Grandjean (2013) pointent les limites des simulations qui n'intègrent pas ou peu les comportements humains. Ces approches utilisent des profils standards d'occupation basés sur une vision élémentaire de la présence/absence dans l'habitat. Ces modèles ont une fonction normative et ne permettent pas de simuler la relation entre comportements et consommations dans l'habitat. Lee, Yi et Malkawi (2011) proposent de dépasser cette limite en s'appuyant sur l'observation des comportements dans le tertiaire pour élaborer un modèle dynamique d'occupation des locaux. Cependant, le tertiaire (vie professionnelle) n'est pas assimilable au monde résidentiel (vie quotidienne). Kashif (2014) a abordé la complexité dans l'habitat en se focalisant sur l'observation et la modélisation de l'activité humaine liée à l'usage de quelques équipements dans la maison (par exemple le réfrigérateur). De notre côté, pour une problématique d'efficience énergétique, nous proposons d'aborder l'activité humaine de vie quotidienne dans son 
ensemble. Plus largement encore, nous pensons qu'il faut considérer l'activité humaine sur de longues durées pour une diversité et multiplicité de populations et d'habitats (Huraux, 2015).

SMACH se situe dans une perspective de conception d'un système d'aide à la décision pour des problématiques d'efficience énergétique (Haradji, Poizat, \& Sempé, 2012). Plusieurs principes sont à la base du développement de la plateforme :

- Il s'agit d'une simulation sociale c'est-à-dire d'une formalisation informatique de processus sociaux (Lefèvre, 2016). Dans notre cas des agents autonomes représentent des acteurs humains qui organisent leurs activités individuelles et collectives dans l'habitat.

- Le modèle de l'activité humaine rend possible des simulations sur des durées variables (une heure, une journée, une semaine, etc.).

- Les modèles d'habitats sont structurés en pièces et ont un comportement thermique qui intègre différents niveaux d'isolation. La température intérieure est donc dépendante de l'isolation de l'habitat et de la température extérieure.

- Les modèles d'appareils représentent une certaine diversité d'équipements possibles dans l'habitat (chauffage, ballon d'eau chaude, réfrigérateur, télévision, ordinateur, lave-linges, lave-vaisselles, lumière, etc.).

Le calcul des consommations d'énergie va donc dépendre du réalisme de l'activité humaine et du réalisme de l'environnement.

La plateforme de simulation SMACH est composée de plusieurs parties (voir schéma cidessous). Le scénario (repère 1 sur le schéma) intègre différentes caractéristiques du foyer (nombre de personnes dans le foyer, âges, sexes, actions possibles, préférences de confort, équipements...) : il a un rôle de cadrage général de la simulation. Le moteur d'activité humaine (repère 2 sur le schéma) va créer la dynamique entre les agents. À chaque pas de temps (correspondant à une minute de simulation) les agents peuvent modifier leurs agissements et interactions avec les autres agents de la situation (les membres du foyer) ou avec l'environnement (l'habitat, le chauffage, etc.). Ainsi, un scénario ouvre sur un ensemble d'actions possibles tandis que le moteur d'activité humaine détermine ce qui sera réellement fait dans la situation simulée. Par exemple, un agent souhaite faire une promenade (action possible dans le scénario) mais un autre agent refuse car il est déjà engagé dans une action. Nous distinguons ainsi dans la simulation ce qui est de l'ordre du cadrage de la simulation (le scénario) et ce qui est de l'ordre de la dynamique des agents (le moteur d'activité humaine).

Figure 1 : Les différents composants de SMACH. Figure : The different components of SMACH

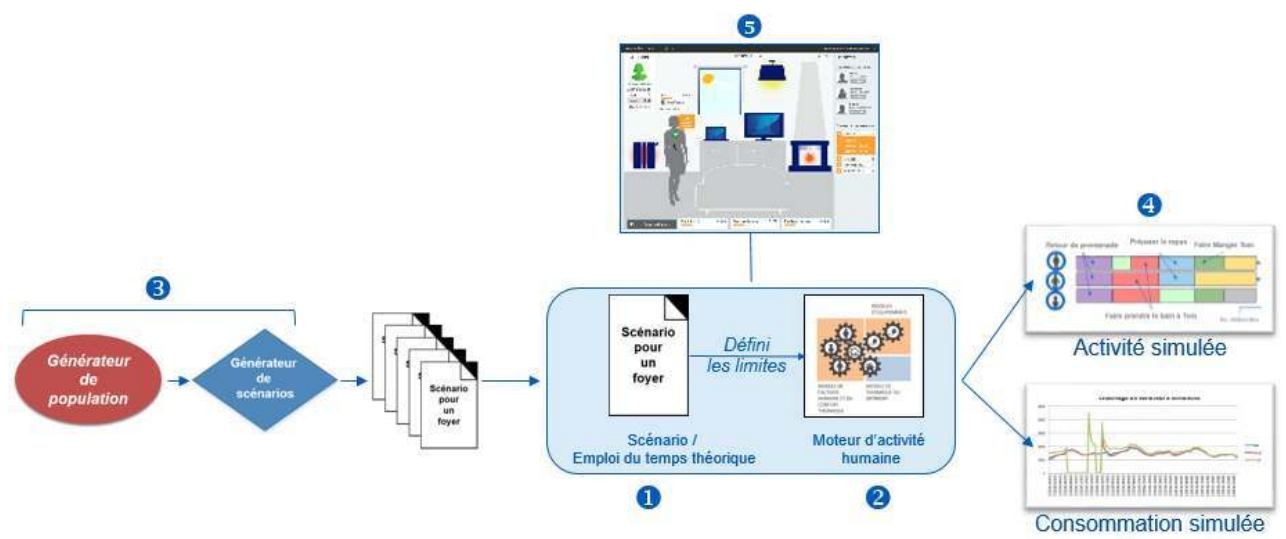


11 Le scénario et le moteur d'activité humaine correspondent à la brique de base de la simulation SMACH (la zone en bleue sur le schéma ci-dessus). Cet environnement technique est complété par des générateurs de population et de scénarios (repère 3 sur le schéma). En effet, il était nécessaire de pouvoir faire des simulations grande échelle pour répondre à des problématiques sur l'efficience énergétique. Par exemple, l'ADEME (Agence De l'Environnement et de la Maitrise de l'Énergie) s'interroge à propos de l'impact des politiques énergétiques sur la courbe des consommations nationales. Le générateur de population (Gargiulo, \& al., 2010) reconstitue une population de foyers (composition des foyers, âges, sexes, types d'habitat, etc.) sur la base des données de recensement de l'INSEE. Les scénarios sont créés en reconstituant des emplois du temps théoriques à partir de l'enquête "emploi du temps"' de l'INSEE (Reynaud, \& al, 2017). Le modélisateur peut alors créer des simulations unitaires (quelques foyers), ou à l'échelle d'une population utiliser les générateurs de population et de scénarios. Il est possible de suivre le déroulé d'une simulation mais ce sont les résultats de la simulation qui sont utiles. Nous obtenons ainsi (repère 4 sur le schéma) le diagramme d'activité et la courbe de consommation pour chaque foyer simulé. Enfin, cet ensemble technique est complété par une interface de simulation participative (repère 5 sur le schéma). Il était important, dans une logique de validation, qu'un acteur humain puisse interagir avec la simulation. Ce dernier peut modifier les actions de son avatar, son niveau d'habillement, les consignes de température et peut allumer ou éteindre tous les équipements de l'habitat.

Dans cet article nous nous focaliserons sur la simulation de l'activité humaine à l'échelle locale d'un foyer (le moteur d'activité). Nous esquisserons une première réflexion sur la simulation grande échelle en conclusion de ce texte. Par contre, nous ne présenterons pas la démarche mise en œuvre pour valider le réalisme des consommations énergétiques. Ce travail sur les consommations a été réalisé (Delenne, 2017 ; Haradji, \& Sempé, 2015) en comparant des courbes de consommation réelles à des courbes simulées comme dans l'exemple présenté ci-dessous.

Figure 2 : Exemple de comparaison (Delenne, 2017) entre une courbe de consommation simulée et une courbe de consommation réelle (1000 foyers, année 2014, semaine 3 , tarif base).

Figure 2: Example of comparison (Delenne, 2017) between a simulated consumption curve and an actual consumption curve (1000 households, year 2014, week 3 , base rate)

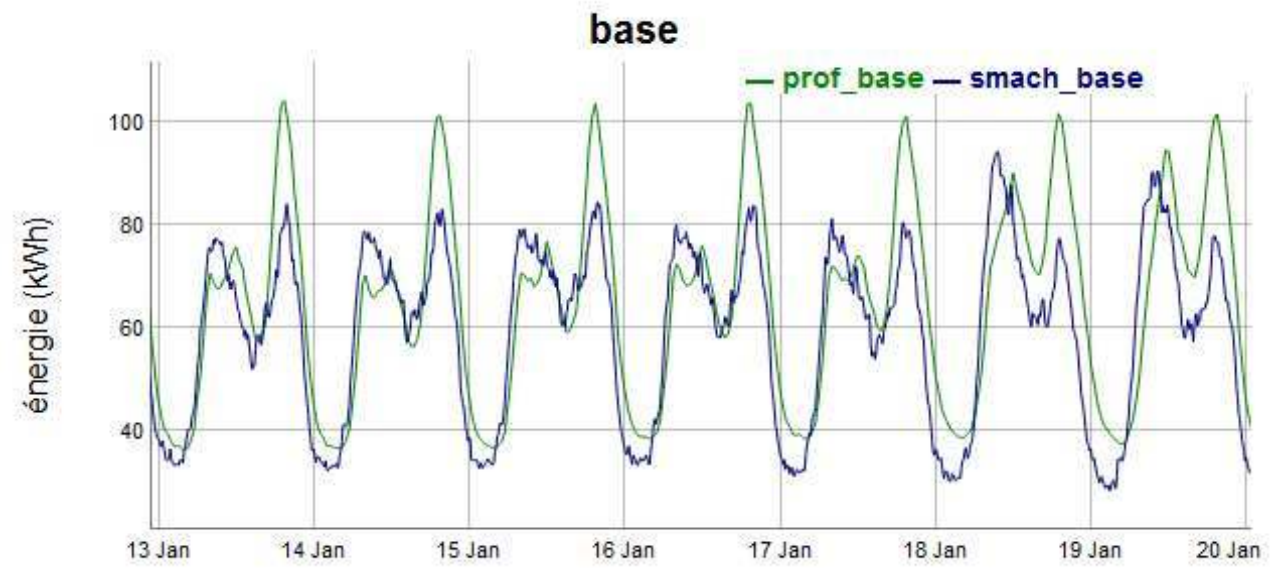

La comparaison des courbes de consommation a permis de valider le réalisme des simulations de consommation. Des experts de différents métiers (thermique du bâtiment, économie, véhicule électrique, autoproduction, efficacité énergétique...) s'appuient 
aujourd'hui sur les données de consommation produites par SMACH et réalisent des études pour des situations futures d'utilisation de l'électricité.

\subsection{Une base empirique pour concevoir le réalisme de l'activité humaine simulée}

Il n'aurait pas été possible de développer cette plateforme de simulation sans l'expérience acquise dans un ensemble de travaux empiriques : sur la gestion d'énergie (Haué, 2004), sur la modélisation des activités humaines (Poizat, Fréjus, \& Haradji, 2009; Guibourdenche, 2013) et sur la caractérisation située des dimensions contextuelles dans l'activité domestique (Salembier, Dugdale, Fréjus, \& Haradji, 2009 ; Guibourdenche, 2013 ; Guibourdenche, Vacherand-Revel, Fréjus, \& Haradji, 2015).

Les résultats de Guibourdenche (2013) sont plus particulièrement mobilisés ici. Ces travaux visaient à caractériser des contextes d'activité domestique de façon à contribuer à la conception de systèmes ambiants efficients énergétiquement. Les analyses ont été construites sur la base des données recueillies par Poizat et al. (2009) auprès de six familles (couples ayant de 1 à 3 enfants). La moitié des familles avait au moins un enfant en bas âge et l'autre moitié au moins un adolescent. Le recueil de données, pour chaque famille, a eu lieu sur trois jours différents (deux jours en semaine et un le week-end), et les enregistrements audio-vidéo portent sur différentes pièces de vie (18 heures d'enregistrement par famille). Des entretiens d'auto-confrontation (Theureau, 2004) ont été menés sur des moments importants de l'activité familiale. Guibourdenche (2013) a proposé d'aborder trois dimensions du contexte de l'activité dans l'habitat : la dynamique individuelle et la signification de l'action pour l'acteur, les articulations collectives de ces significations et les agencements physiques de l'activité. Il a ainsi pu caractériser des contextes d'activité dans lesquelles les effets de consommations d'électricité émergent en lien à des contraintes physiques dans l'habitat et à la signification de l'action pour les habitants.

16 Sur ces bases, nous présentons dans les sections suivantes les choix de conception réalisés et nous les discutons en les confrontant aux éléments de connaissance issus des travaux de Guibourdenche (2013) sur l'activité domestique en situation naturelle.

\section{L'approche énactive de l'activité humaine pour la simulation : hypothèses de l'activité individuelle, collective et du couplage à l'environnement}

Le point de départ structurant pour concevoir le réalisme de la simulation repose sur nos postulats théoriques liés à l'activité humaine. Nous considérons ci-dessous trois hypothèses théoriques issues de l'énaction (Maturana, \& Varela, 1994 ; Varela, 1989) et du cours d'action (Theureau, 2015). Elles nous ont directement inspirés pour le cadrage initial de la modélisation de l'activité simulée. Cette perspective énactive est particulièrement relayée dans la littérature en Intelligence Artificielle. L'ouvrage de Winograd et Flores (1989) a notamment fait date en proposant une refondation de la conception informatique à partir du paradigme de l'énaction.

18 La première hypothèse est relative à l'autonomie des systèmes vivants et se nomme hypothèse de l'autopoïèse par Maturala et Varela (1994). Tout système vivant constitue 
un système autonome qui doit maintenir son équilibre (sa clôture opérationnelle) dans la dynamique de ses interactions avec l'environnement (la dynamique de son couplage structurel). La convergence entre l'énaction et les SMA (Système Multi-Agent) porte principalement sur l'autonomie des entités. Nous ne parlons pas d'autopoï̀se pour les agents mais bien d'autonomie des agents dans la simulation. L'énaction propose une distinction forte entre unités vivantes et machines autonomes: les machines sont incapables de générer les composants de leurs propres organisations, là où les unités vivantes le sont. SMACH est un système essentiellement réactif dans lequel l'autonomie se décline principalement par l'émergence d'une organisation familiale, non définie par le modélisateur, mais résultant de l'ensemble des interactions locales à chaque pas de temps. L'observateur de la simulation constate alors les prises de décisions de chaque agent et l'évolution d'une organisation de vie quotidienne.

La deuxième hypothèse est relative à l'acteur humain qui détermine son engagement dans une situation en fonction de son histoire et de la situation dans laquelle il se trouve. Il transforme son environnement et se transforme dans la dynamique de ses interactions. Cette interaction est asymétrique car c'est l'acteur qui définit ce qui est significatif/ pertinent pour lui dans son environnement. Le système artificiel de son côté ne vise qu'une imitation de surface de la logique du vivant. Il ne vise donc aucune réalité cognitive. Il ne peut pas en effet être question d'une création de signification par des agents artificiels. Ce sont les règles définies par le modélisateur qui permettent à l'agent de réagir dans l'interaction. Pour cela, nous avons utilisé un modèle individu centré (Phan, \& Amblard, 2007) qui favorise la construction d'une dynamique numérique spécifique au point de vue de chaque agent (une personne correspond à un agent et une famille à un ensemble d'agents). Cette imitation de surface est alors portée par le couplage asymétrique de chaque agent avec son environnement et par les mécanismes de coopération (communication) des agents entre eux.

Enfin, avec la troisième hypothèse, à la suite de Theureau (2004), nous considérons l'activité collective comme une totalité dont l'organisation est constamment remise en cause par les activités individuelles et constamment reconstruite par ces mêmes activités individuelles. Le collectif n'est donc pas une unité autonome car il correspond à une « totalité » constamment « détotalisée » et « retotalisée » par l'activité de ses composants. Le système artificiel, selon cette logique, construit l'activité collective à partir des activités individuelles mais la déconstruit aussi à partir des objectifs individuels de chaque agent. Dans la simulation SMACH c'est l'autonomie des agents engagés dans l'interaction qui rend possible cette activité collective.

21 Ce sont ces concepts relatifs à la description de l'activité humaine qui sont à la base de nos hypothèses pour la modélisation de l'activité humaine simulée. Nous avons alors distingué 1) l'autonomie et la dynamique de l'activité individuelle de l'agent; 2) la construction de l'activité collective d'un agent avec d'autres agents; 3) le couplage asymétrique de l'agent avec son environnement. Huraux, Sabouret et Haradji (2014) et Huraux (2015) s'appuient sur ces trois hypothèses pour proposer un modèle général de la simulation (voir la figure 3 ci-dessous). Sans entrer dans le détail de ce modèle, nous identifions ci-dessous comment ces hypothèses structurent la modélisation multi-agent (les éléments du modèle sont en italique dans le texte) :

- L'activité individuelle est portée par un agent (un individu) qui réalise de façon autonome des tâches. Des habitudes émergent au cours de la simulation et elles sont influencées par des modes de vie liés à l'efficience énergétique, au confort, au budget. 
- L'activité collective se construit dans l'interaction entre agents (les individus) qui réalisent ensemble des tâches et forment pour l'occasion des groupes éphémères composés de tout ou partie des membres du foyer (adultes, enfants, proches...).

- Le couplage structurel avec l'environnement résulte des agents (les individus) qui, engagés dans leurs activités individuelles et collectives, sont en interaction avec un environnement composé d'appareils (chauffage, ballon d'eau chaude, réfrigérateur, etc.) et de pièces qui forment un logement ayant un niveau d'isolation. De plus le fournisseur propose des offres tarifaires.

Figure 3 : L'organisation multi-niveau de l'activité humaine avec les relations d'influence (flèches bleues en pointillés) et d'interaction (flèches noires) entre entités du modèle (Huraux, 2015). Figure 3: Multi-level organization of human activity with relations of influence (blue dotted arrow) and interaction (black arrow) between model entities (Huraux, 2015)

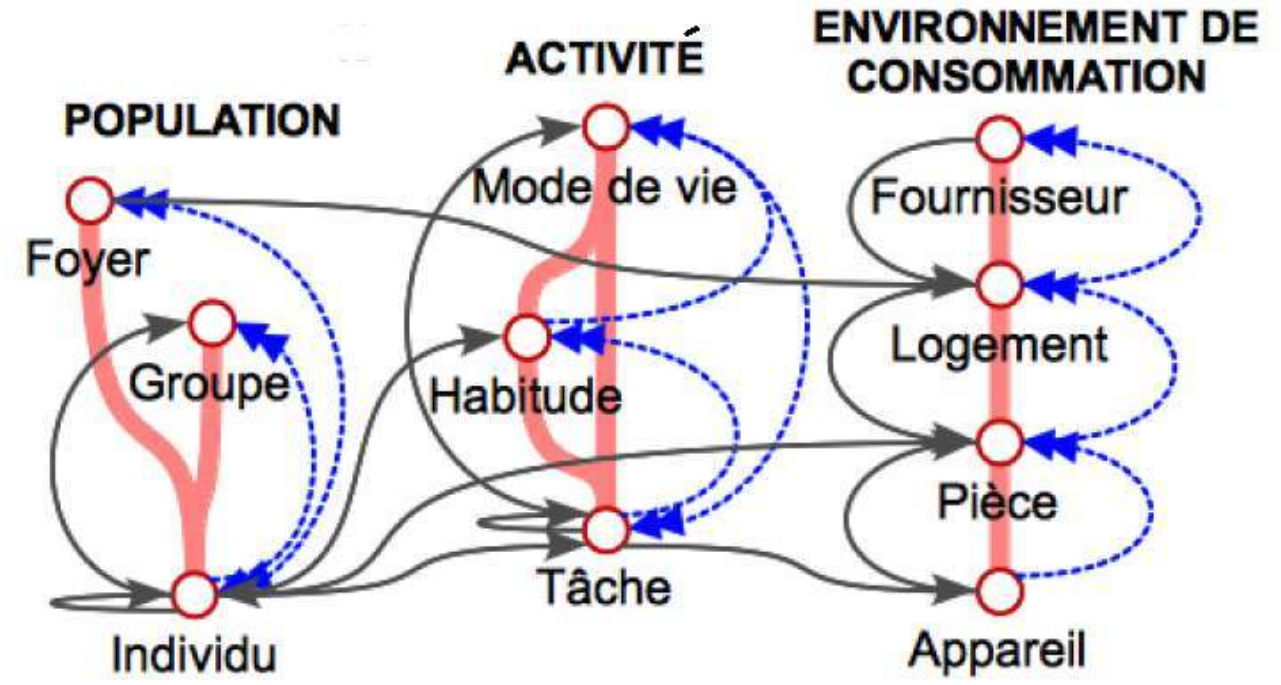

Les hypothèses de l'énaction et du cours d'action sont ici déterminantes pour cadrer le modèle et définir les bases du réalisme de la simulation. Nous organisons les trois sections suivantes de cet article autour de ces trois hypothèses épistémologiques et nous explicitons pour chacune d'elles des proximités et des différences entre les connaissances issues de l'analyse empirique et les dynamiques créées dans la simulation.

\section{Analyse empirique et simulation des dynamiques individuelles de l'activité humaine}

\subsection{Contexte multi-préoccupationnel et séquences d'action}

Guibourdenche (2013) caractérise globalement l'activité humaine dans l'habitat comme un « contexte multi-préoccupationnel » : enchaînement ou conjonction dans les actions, anticipation ou historicité des actions, interruption dans l'activité etc. Il caractérise, par exemple, une organisation de soirées structurées autour d'une alternance de préoccupations qui peuvent être suspendues, ouvertes, effectuées en partie ou en totalité (Figure 4). À la suite d'un appel d'un des enfants, un parent peut suspendre momentanément une activité de loisir pour s'occuper de l'enfant, puis revenir à son occupation antérieure. À d'autres moments ce sont des évènements liés aux appareils qui 
vont ouvrir sur des basculements contextuels, comme des sonneries de téléphones et de fours, des fins d'émissions télévisées. Enfin, dans l'activité des habitants, les préoccupations émergent également de façon conjointe à certains moments (voir figure 4 ci-dessous). Les habitants vont par exemple mener deux actions de front, parfois un peu plus, comme suivre un feuilleton télévisé et réaliser le repassage.

Figure 4 : Contexte multi-préoccupationnel dans l'activité quotidienne (Guibourdenche, 2013). Figure 4: Multi-concern context in daily activity (Guibourdenche, 2013)

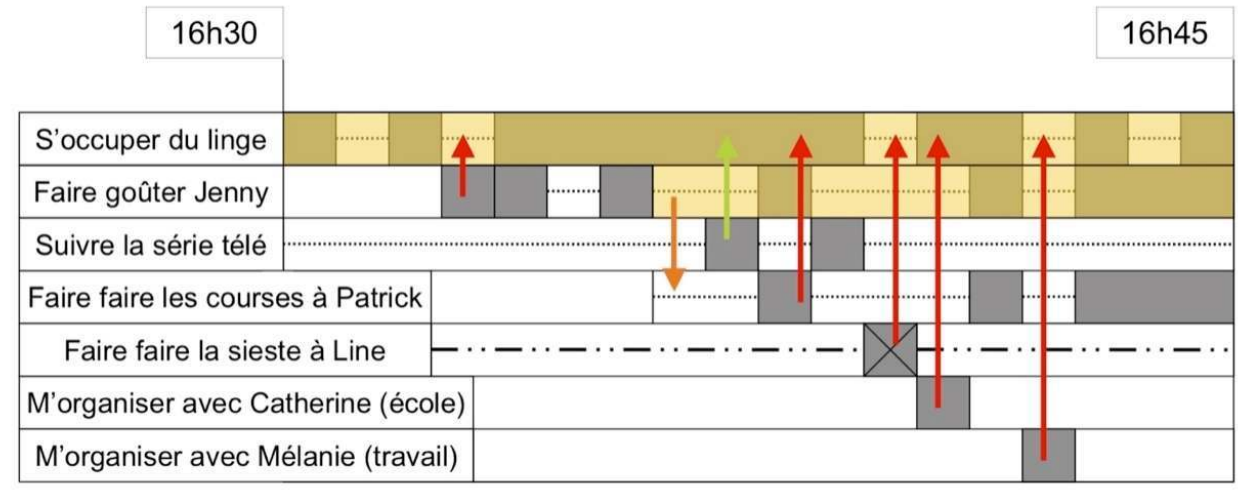
point de vue des habitants. Certaines en agrémentent d'autres (e.g., suivre un feuilleton télévisé en repassant, regarder la télévision en patientant avant le coucher des enfants...). Certaines en empêchent ou gênent d'autres, leur font concurrence. Par exemple, faire des courses, repasser et suivre un feuilleton télévisé sont relativement difficiles à actualiser de front. D'autres, sont en relation de nécessité. Ainsi, pour pouvoir passer une soirée dans le salon, les parents ont à un moment besoin de coucher leurs deux enfants. Les événements considérés par les habitants ne sont pas uniquement contextuels de la préoccupation qui les circonscrit. Ils le sont également d'une partie des autres préoccupations (nécessité, concurrence ou agrément).

La dynamique de l'activité de chaque agent s'approche en partie d'une activité humaine individuelle. Les actions sont organisées selon une logique qui est propre à la situation de chaque agent. Elles peuvent être interrompues et reprises mais aussi déplacées ou abandonnées. La figure 5 ci-dessous correspond à un extrait d'une soirée et va nous permettre d'évoquer l'intérêt et les limites de la modélisation pour cette simulation. 
Figure 5 : Extrait d'une soirée dans une simulation d'une semaine avec SMACH.

Figure 5 : Extract from an evening in a week-long simulation with SMACH

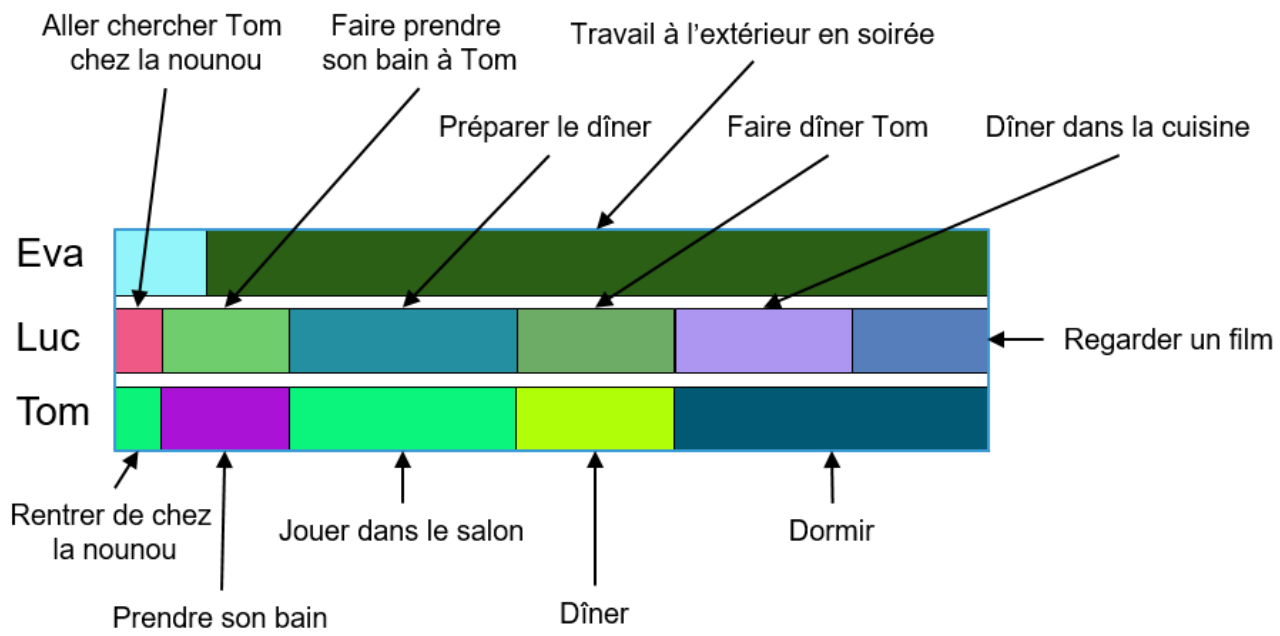

Chaque ligne horizontale correspond à un agent et chaque couleur à une action : chaque agent a une dynamique d'action qui lui est propre. Plus précisément, l'agent-Luc (le père) va enchaîner un ensemble d'actions visant à s'occuper de l'agent-Tom (son fils en bas âge) et à organiser ensuite sa soirée (repas et télévision). Par contre, comme on peut l'entrevoir avec cet exemple, un agent ne gère pas plusieurs actions dans une même unité de temps (pas de conjonction de préoccupations).

La modélisation pour la simulation produit cette description séquentielle de l'action pour éviter une démultiplication trop grande des actions possibles et de leurs états. Cette simplification visant à éviter un système ingérable est le résultat d'un compromis considéré comme acceptable vis-à-vis de la simulation de consommations d'électricité. En effet, dans la simulation, comme dans la vie réelle, les appareils peuvent fonctionner en même temps que les agents/habitants réalisent des actions. Ainsi, un agent peut démarrer une machine à laver le linge et réaliser ensuite d'autres actions pendant qu'elle fonctionne. Il peut également regarder la télévision (TV), s'interrompre sans l'éteindre, revenir la regarder ou même la laisser en tant que fond sonore et visuel. Un agent ne peut donc pas mener plusieurs actions de front mais il peut utiliser plusieurs équipements électriques dans un même temps car une action peut déclencher plusieurs équipements. Un agent peut alors repasser du linge (une seule action) avec deux appareils en fonctionnement (fer à repasser et TV). L'objectif de calcul des consommations détermine ici le caractère acceptable d'une réduction de l'activité humaine réelle.

\subsection{Les régularités et variabilités temporelles d'action}

Les analyses empiriques montrent qu'il existe des régularités dans l'activité de vie quotidienne. Guibourdenche (2013) décrit par exemple des régularités de préoccupations significatives liées au coucher des enfants entre plusieurs soirées et après-midi. Par ailleurs les préoccupations de l'activité sont multiples et ont des temporalités très diverses (de quelques minutes à plusieurs années). Ainsi, les repas reviennent régulièrement dans la journée et d'un jour sur l'autre. Le traitement du linge (centraliser le linge sale, laver, sécher, repasser) est une préoccupation régulière sur la semaine et tout au long de l'année tandis que certains travaux n'ont pas de régularité mais peuvent 
se dérouler sur de longues périodes. Par exemple les travaux de rénovation du bâtiment décrits par Guibourdenche (2013) structurent l'activité quotidienne régulière de la famille durant plusieurs semaines, mais ils ne le font qu'une seule fois dans la vie de ce foyer, donnant lieu à de multiples préoccupations et enjeux de coordination et de priorisation. Cette émergence est toujours située dans un contexte particulier pour l'habitant à l'instant « $t$ », ce qui donne un sens local spécifique à l'activité en cours. Faire dormir un enfant peut signifier localement: de le coucher pour la nuit, de lui faire faire la sieste pour qu'il soit en forme durant l'après-midi, ou de lui faire faire la sieste pour qu'il récupère d'une après-midi active.

La simulation multi-agent reprend cette forme d'organisation de l'activité humaine. Les comportements des agents sont ainsi envisagés sur différentes échelles de régularité/ variabilité : sur une journée (dormir, manger...), sur une semaine (entretenir le linge, faire le ménage...) ou toute autre périodicité (par exemple manger au restaurant environ une fois par mois). Simuler cette dimension temporelle générique de variabilité/ régularité de l'activité humaine rend la notion de rythme indispensable pour générer des simulations sur une longue période. Un rythme va alors s'appliquer à une action, avec une certaine variabilité, une fréquence et une période d'application.

Figure 6 : Exemple de réalisation non-déterministe des actions dormir (violet) et laver du linge (orange) sur le plan temporel (en abscisses les jours sur un mois et en ordonnées les heures de la journée).

Figure 6: Example of non-deterministic realization of the actions sleep (purple) and wash laundry (orange) on the temporal plane (in abscissa the days over a month and in ordinate the hours of the day)

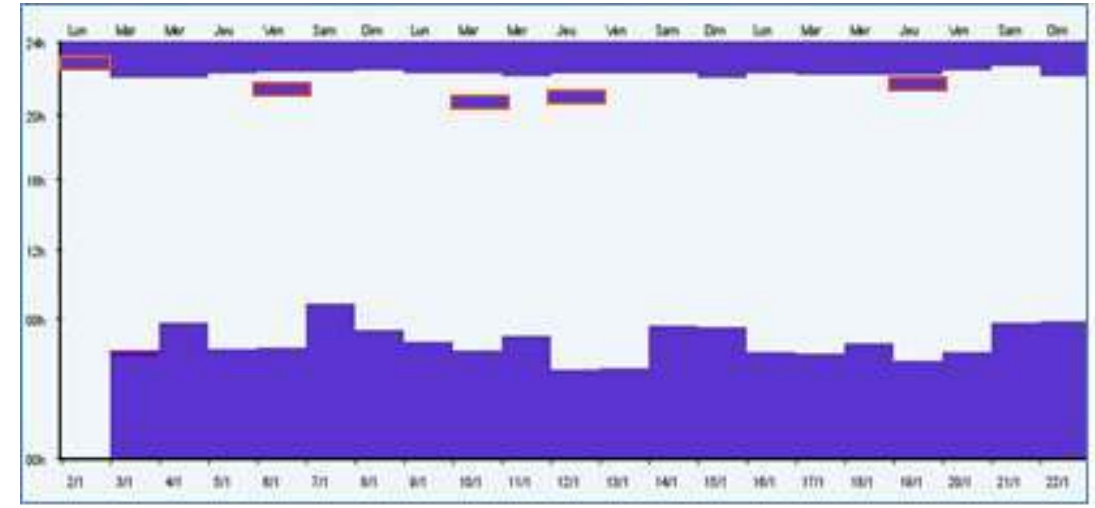

30 La figure 6 ci-dessus illustre la dimension non-déterministe de la simulation. Dans cet exemple, nous avons sélectionné sur un mois deux actions réalisées par l'agent-John (dormir et laver du linge). L'action dormir débute vers 23 heures et se termine vers 6 heures en semaine et vers 8 heures le week-end. Nous pouvons distinguer cette régularité dans le sommeil mais nous pouvons aussi constater que l'agent-John se lève à des heures variables en semaine et le week-end. Nous constatons qu'il se lève aussi plus tard le mercredi, notamment car il ne travaille pas ce jour-là. L'action de laver le linge (les traits horizontaux isolés) est répartie dans la semaine, plutôt le soir.

Le comportement simulé d'un agent repose sur la décision qu'il prend en fonction des actions qu'il peut réaliser, de ses préférences, d'un ensemble d'habitudes et de son couplage à la situation. C'est le modélisateur qui a préalablement défini les caractéristiques générales de l'emploi du temps (le scénario) mais c'est l'agent qui agira en fonction de son couplage à la situation. L'agent dispose donc d'une certaine autonomie 
et, d'une simulation à l'autre, il n'y a pas tout à fait les mêmes comportements aux mêmes horaires, ou pas tout à fait dans le même ordre. Certaines formes d'organisation peuvent aussi émerger du déroulement de la simulation. Le système peut identifier des configurations d'actions qui respectent bien les contraintes qu'il doit gérer (préférences, rythmes) et qu'il cherchera alors à répéter. Il crée alors de nouvelles habitudes.

Il y a donc bien des régularités et des variabilités dans la simulation qui sont approchantes d'un comportement de surface de l'activité humaine. L'autonomie relative dont disposent les agents s'inscrit principalement dans le cadrage réalisé par le modélisateur sur la situation à simuler.

\section{Analyse empirique et simulation des dynamiques collectives de l'activité humaine}

L'analyse empirique des situations domestiques permet de caractériser l'articulation collective des activités individuelles. Cette articulation a été décrite par Guibourdenche (2013) sur un continuum allant de différences à similarités quasi-complètes des préoccupations des habitants (voir figure 7). Par exemple, le moment du repas dans les foyers observés correspond très souvent à une articulation des préoccupations à similarité quasi-complète, car tous les acteurs ont des préoccupations thématiquement très proches. Inversement, il peut y avoir une articulation collective faible quand deux acteurs ont des préoccupations similaires alors que trois autres ont des préoccupations respectivement différentes (e.g., contexte $\mathrm{n}^{\circ} 2$ sur la figure 7 ). Ce peut être le cas quand deux personnes préparent un repas quand d'autres vaquent à leurs occupations respectives.

Figure 7 : Possibilités génériques d'émergence des contextes d'articulation collective pour cinq acteurs (Guibourdenche, 2013). Les couleurs vertes indiquent des similarités entre les préoccupations, le blanc indique des préoccupations différentes pour chaque acteur.

Figure 7: Generic possibilities for the emergence of collective articulation contexts for five actors (Guibourdenche, 2013). Green colors indicate similarities between concerns, white indicate different concerns for each actor

\begin{tabular}{|c|c|c|c|c|c|c|}
\hline & Acteurs $\mathbf{N}^{\circ}$ : & 1 & 2 & 3 & 4 & 5 \\
\hline \multirow{7}{*}{ 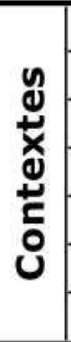 } & 1.Différence quasi-complète & & & & & \\
\hline & 2. Similarité à 2 & & & & & \\
\hline & 3. Similarité à 3 & & & & & \\
\hline & 4. Similarité à 4 & & & & & \\
\hline & 5. Doubles paires de similarité & & & & & \\
\hline & 6. Paire et triade de similarité & & & & & \\
\hline & 7. Similarité quasi-complète & & & & & \\
\hline
\end{tabular}

La simulation rend compte de ces distinctions et les traduit sous forme de coopération entre agents. Huraux (2015) définit un concept de groupes éphémères d'agents c'est-àdire de collectifs éphémères d'agents qui évoluent au fur et à mesure de la situation et des besoins des agents. Ce concept se rapproche assez bien de ce que nous observons dans la vie quotidienne réelle au plan des articulations collectives comme sur le plan de l'occupation de l'espace. Ce sont des règles de type «aider à » qui vont permettre à un agent adulte d'aider un agent-enfant ou des règles de type "obligatoirement ensemble » ou « plutôt à plusieurs » qui feront que des agents mangeront ensemble ou regarderont la 
TV ensemble. La simulation rend ainsi possible des actions collectives mais cette coordination n'est pas déterministe. La coopération entre agents est construite à partir de prises d'information dans l'environnement (voir qu'un agent-enfant est habillé) et d'actes de langage (demande d'information, demande d'action, réponse, etc.).

Figure 8 : Extrait d'une articulation collective d'activités individuelles. Les couleurs identiques indiquent des similarités entre les activités.

Figure 8: Collective articulation of individual activities. Identical colors indicate similarities between activities

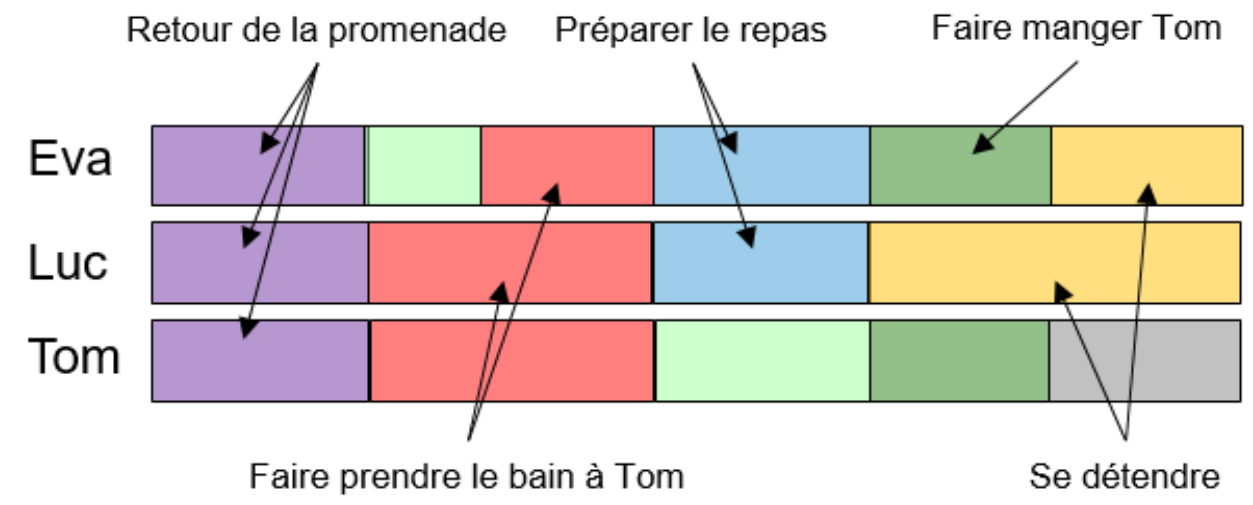

La figure 8 présente une articulation collective simulée. Plusieurs collectifs éphémères émergent entre le retour de promenade (trois personnes), la préparation du repas (les parents), le repas de l'enfant (la mère et l'enfant) ou encore le moment de détente (les parents).

Cependant, l'activité collective telle qu'elle est simulée ne peut prétendre au niveau de finesse observée en situation réelle. Par exemple, Guibourdenche (2013) décrit l'évolution de l'organisation à travers le temps entre divergence et convergence d'acteurs dans le besoin d'articuler les préoccupations de repassage, de listes des courses à faire, de travaux de rénovation à vérifier, de goûter de l'enfant etc. Cette finesse dans le calage progressif des actions et préoccupations entre acteurs est impossible dans ce type de simulation. Cette finesse de description de l'activité collective n'est pour l'instant pas considérée comme nécessaire pour évaluer la relation entre consommation de l'énergie et activité.

\section{Analyse empirique et simulation du couplage de l'activité à l'environnement}

Guibourdenche (2013), dans la perspective de conception d'un système de gestion d'énergie couplé à la vie familiale, a caractérisé les contextes physiques dans lesquels les équipements électriques sont ou non utilisés pour pouvoir définir quand les couper ou non, quand réguler et comment. Il a pour cela articulé l'analyse de l'activité individuelle et collective à celle des (Im)possibilités d'action et de cognition du fait de l'agencement de l'habitat et des équipements. Certains contextes sont simples : des habitants suivent leur émission télévisée devant leur téléviseur dans le salon... Mais la plupart sont beaucoup plus complexes. Ainsi, un habitant peut suivre une série télévisée diffusée sur sa télévision du salon, alors qu'il/elle se trouve dans la cuisine. À d'autres instants, un individu se trouvant face à la télévision ne suit pas le feuilleton. À d'autres moments enfin, le téléviseur est laissé en marche alors qu'il n'est pas possible de la regarder 
(téléviseur et habitants sont physiquement dans des étages différents, il y a du bruit près des habitants...). Cette analyse permet aussi de mettre en évidence les différentes ressources et contraintes de la communication entre les habitants d'une maison au quotidien (e.g., les bruits, les paroles...), celles de l'occupation de l'espace physique (e.g., occuper une salle de bain, un espace de repassage) et enfin l'état de marche des appareils.

La question du couplage des agents à l'environnement est une question importante pour la simulation. Tandis que Guibourdenche considère l'impact de l'agencement physique de l'espace sur l'activité (problématique de l'internet des objets) nous devons, pour la simulation, 1) créer les ressources et contraintes de l'environnement et 2) définir l'impact de l'activité humaine simulée sur l'environnement. Il a donc été nécessaire pour cela d'intégrer un modèle de bâtiment et de son environnement (voir Plessis, \& al, 2014). Cette modélisation comprend une description de l'habitat (pièces, type d'isolation, fenêtres etc.), une description des appareils électriques et de leur consommation (réfrigérateur, chauffage...) ainsi qu'une description de l'environnement climatique extérieur (données issues de sources météorologiques). Cette description rend possible une dynamique simple de couplage des agents avec l'environnement. Ils vont ainsi :

- Réaliser leurs actions dans des pièces selon des contextes simples (un agent regarde la télévision dans la salle à manger, dort dans sa chambre, etc.).

- Agir et déclencher le fonctionnement d'un équipement (télévision, ordinateur, ballon d'eau chaude, chauffage, etc.). Par exemple, le fonctionnement du ballon d'eau chaude est directement lié au fait de prendre un bain ou une douche (quantité d'eau différente) et aux visées d'économie liée au tarif (fonctionnement de nuit ou de jour). Il en est de même pour le réfrigérateur qui aura une consommation plus importante au moment des repas (ouverture/fermeture de la porte du réfrigérateur).

- Utiliser des informations de l'environnement pour se coordonner. Une pièce peut être occupée et ne pas être disponible ou bien encore un agent A peut préparer un petit déjeuner et dans ce cas l'agent $B$ le « verra » et n'aura pas à le faire.

Mais cette dynamique de couplage n'est pas suffisante dans le cadre de notre visée de conception sur l'efficience énergétique. Nous avons dû tenir compte du confort de l'habitant et des actions qu'il peut réaliser pour le préserver ou l'améliorer (Plessis, Amouroux, \& Haradji, 2014). Pour cela, nous avons défini un modèle décisionnel du confort de chaque agent qui s'appuie sur le modèle de Fanger (1970). Il fonctionne ainsi :

- Chaque agent a une perception relative du confort. Cela permet de quantifier le confort thermique ressenti en proposant un pourcentage d'insatisfaction qui est fonction de la température de la pièce, de l'habillement et de l'action réalisée par l'agent. Par exemple un agent passant l'aspirateur dans une pièce avec un habillement léger pourra avoir chaud alors qu'un autre agent, utilisant un ordinateur dans la même pièce, pourra avoir froid.

- Les agents vont chercher à préserver leur confort. Le calcul d'une insatisfaction va inciter l'agent à modifier son environnement. En fonction de l'insatisfaction l'agent pourra augmenter/diminuer une température de chauffage, augmenter/diminuer son habillement, fermer/ouvrir une fenêtre et changer ou pas d'activité. Quand plusieurs agents sont dans la même pièce les agents les moins nombreux qui ont trop chaud/trop froid ajustent leur confort thermique en modifiant leur habillement.

Le couplage à l'environnement dans la simulation se distingue en partie de celui proposé dans la description empirique de l'activité. Cette différence résulte pour beaucoup de la visée de calcul de SMACH sur l'efficacité énergétique: un agent-humain sensible au 
confort thermique et en interaction avec un environnement consommateur d'énergie est un point central pour notre problématique de conception.

\section{Confronter le réalisme de la simulation à la situation réelle}

41 Les quatre sections précédentes nous ont permis de situer le réalisme de la simulation par rapport à l'activité humaine. Avec cette dernière section nous souhaitons rendre compte de la validité écologique de la simulation (Béguin, \& Weill-Fassina, 1997). Nous présentons dans cette section quelques principes méthodologiques et quelques résultats de l'expérimentation réalisée.

Nous avons respecté trois principes pour évaluer le réalisme de la simulation:

- Bénéficier d'une situation réelle de référence sur les consommations d'électricité permettant la comparaison avec la simulation. EDF réalise différentes expérimentations en situation réelle. L'expérimentation «Une Bretagne d'avance» a été choisie car disposant de données quantitatives sur la consommation d'environ 500 familles.

- Confronter les membres d'une famille à la simulation de leur vie quotidienne. Pour évaluer le réalisme de la simulation, nous avons impliqué des familles à l'aide de la simulation participative (voir figure 9) et les avons confrontées à la simulation de leur vie quotidienne.

- Articuler validations qualitative et quantitative. Nous avons souhaité valider la simulation sous un angle qualitatif (l'activité dans l'habitat) mais également sous un angle quantitatif ${ }^{3}$.

Nous avons dû créer des conditions d'expérimentation satisfaisantes pour pouvoir confronter des familles à la simulation de leur vie quotidienne, 1) en développant une interface de simulation participative rendant possible la manipulation d'un avatar par un acteur humain qui peut alors modifier le cours de la simulation (figure 9) ;2) en réalisant des entretiens préalables auprès de dix familles issues de l'expérimentation «Une Bretagne d'avance » afin d'être en mesure de réaliser une simulation d'une semaine pour chacune des dix familles; 3) en confrontant un acteur humain de la famille à sa dynamique de vie quotidienne in silico, mais aussi à la dynamique des interactions avec les autres agents de sa famille, afin qu'il décrive et commente cette activité «virtuelle » en lien avec son activité réelle. 
Figure 9: Conception d'un environnement réaliste pour la simulation participative.

Figure 9: Designing a realistic environment for participatory simulation

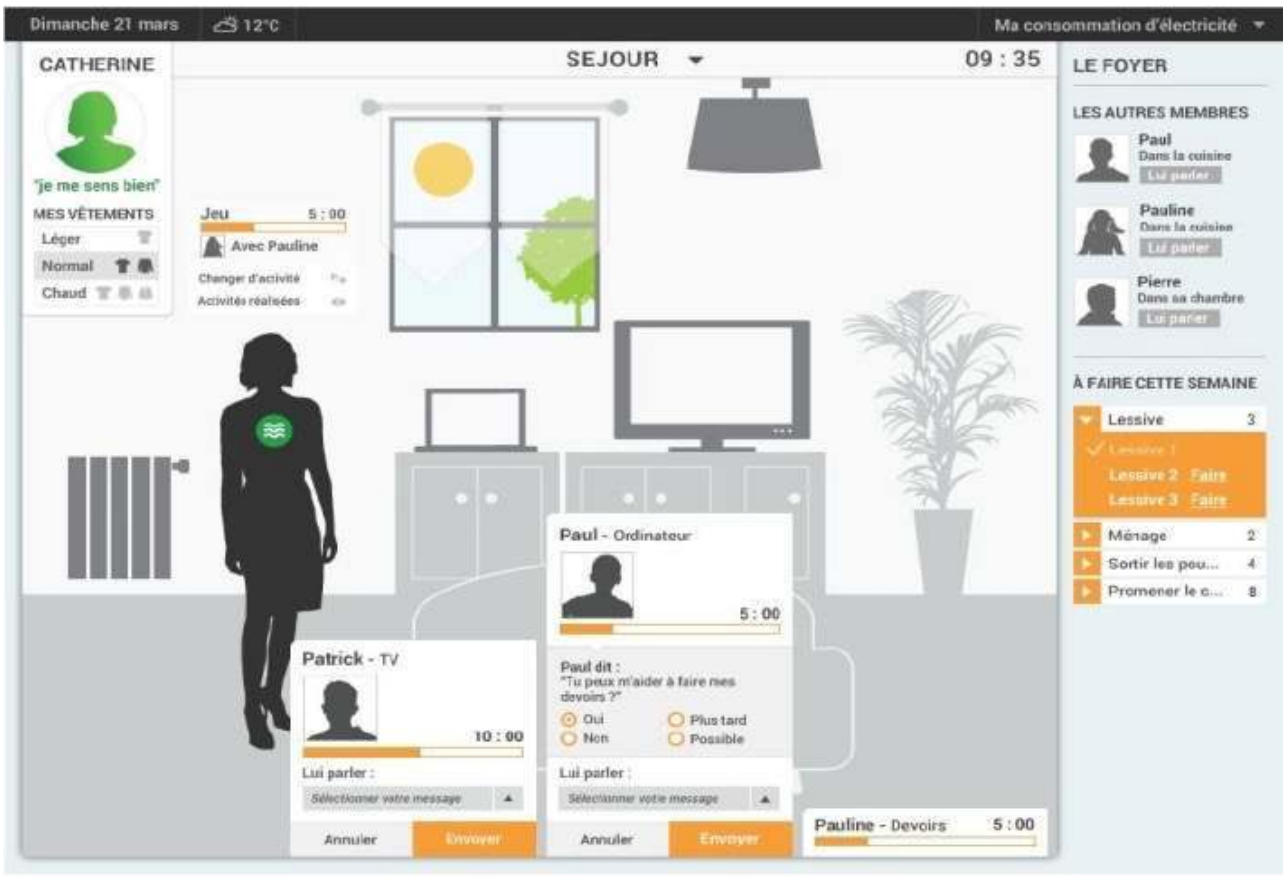

La simulation participative a ainsi permis de mettre en évidence les points suivants :

- Le principe général de la simulation relatif à l'organisation de la vie quotidienne et sa dynamique est bien compris. Le lien avec les activités quotidiennes est considéré comme pertinent.

- L'acteur humain interagissant avec la simulation comprend le point de vue que porte son avatar et fait bien la différence avec l'engagement des autres avatars représentant les autres personnes du foyer. L'exemple présenté à la figure 9 illustre le fait que l'agent Catherine joue avec l'agent Pauline tandis que deux autres agents sont présents dans la même pièce mais sont occupés à d'autres activités (TV et ordinateur).

- La constitution des collectifs et leurs dynamiques d'évolution sont considérées comme réalistes par l'acteur humain. Dans l'exemple de la figure 9 les agents mère et fille jouent ensemble, mais, plus tard dans la simulation, elles auront des activités séparées ou associées à d'autres agents.

- Les habitudes de vie liées au travail, à l'école, au coucher des enfants etc. sont des éléments de la simulation qui agissent comme des jalons significatifs pour l'acteur humain impliqué dans la simulation participative.

- L'interaction entre l'agent et l'environnement physique (radiateurs, fenêtres, vêtements, lumières) ou thermique (température dans la pièce ou température à l'extérieur) crée une expérience de simulation reproduisant des visées de confort ou d'économie d'énergie. La modélisation de l'état de confort de l'agent (la figure 9 montre en exemple l'agent Catherine qui a un confort satisfaisant) joue un rôle de repère permettant à l'acteur humain de se situer dans l'environnement simulé.

Il y a bien sûr des limites méthodologiques à cette expérimentation. Par exemple, nous avons confronté chaque participant à une semaine de sa vie quotidienne. Ce faisant, le participant a tendance à se focaliser sur les régularités de vie et à moins considérer la variabilité de la vie quotidienne. Il pouvait aussi être gêné par son avatar dont il ne 
pouvait pas anticiper l'action suivante. Mais la simulation a parfois aussi permis d'aller au-delà de ce que les familles avaient spontanément indiqué, ouvrant de ce fait sur une explicitation plus approfondie de leur activité. C'est par exemple le cas d'une personne qui nous a précisé faire la sieste quand elle travaillait à la maison.

Les résultats de l'expérimentation sont concluants. Aucun des mécanismes de base de la simulation (autonomie des agents, dynamique individuelle et collective, couplage avec l'environnement) n'a été remis en question. De plus, certaines actions peu cohérentes (un jeune qui va se coucher tous les samedi soir à 22 heures) ou manquantes (l'exemple de la sieste cité précédemment) ont pu être identifiées et modifiées facilement dans le scénario correspondant.

\section{Conclusion et perspectives}

47 Notre proposition, dans cet article, est de concevoir le réalisme de la simulation en respectant une certaine complexité de l'activité humaine. Une simulation SMACH est aujourd'hui le résultat d'une activité individuelle et collective d'agents informatiques, qui organisent leur vie quotidienne dans un habitat numérique, qui génèrent des consommations d'électricité du fait de leurs actions quotidiennes avec des appareils électriques, de leur perception du confort et d'un souci plus ou moins marqué d'efficacité énergétique.

Nous avons présenté les deux niveaux de modélisation qui portent le réalisme de la simulation. Le premier niveau a consisté à cadrer la conception avec les hypothèses épistémologiques de l'énaction et du cours d'action. Le deuxième niveau a consisté à décliner ses hypothèses sur la base de notre connaissance empirique de l'activité dans l'habitat. Les réductions opérées dans la modélisation de l'activité simulée sont considérées comme valides tant qu'elles ne remettent pas en question: A) l'activité humaine et ses effets induits sur la consommation; B) l'objectif de calcul de l'efficience énergétique. Le réalisme de l'activité est ici subordonné à notre objectif de conception et, pour cela, nous n'avons pas recherché le réalisme dans toutes ses dimensions (culturelle, émotionnelle, cognitive) mais plutôt un réalisme suffisant pour la visée de calcul qui est la nôtre.

Cet article a permis d'aborder la question du réalisme de l'activité simulée portée par la simulation. Nos travaux récents, comme nous l'avons indiqué (section 2.2), nous ont permis de réaliser des simulations à l'échelle d'une ville ou d'un pays en articulant un niveau local d'activité (le moteur d'activité) avec des modules de génération de population/de scénarios. Ce pas supplémentaire vers de la simulation grande échelle induit un élargissement dans notre questionnement sur le réalisme des simulations. Nous nous interrogeons ci-après : 1) sur l'articulation de méthodes statistiques à la simulation multi-agent ; 2) sur l'articulation entre un niveau local de l'activité et un niveau supérieur correspondant à une dynamique sociale ; 3) sur l'articulation entre simulation et living lab pour répondre à une problématique d'efficience énergétique.

Pour créer des simulations réalistes à grande échelle nous avons articulé deux méthodes (Reynaud, \& al., 2017). La méthode statistique aboutit à créer des scénarios qui sont valides à un niveau agrégé (par exemple en retrouvant une distribution similaire des activités dans les scénarios et dans les enquêtes emploi du temps) mais elle ne permet pas localement de simuler des individus autonomes, réactifs à leur environnement, capables 
de se projeter dans une situation fictive. La méthode agent, de son coté, dispose de modèles centrés sur les individus et vise un réalisme de vie quotidienne dans l'habitat. La simulation grande échelles permet ainsi de coupler la méthode statistique, basée sur des modèles visant un réalisme statistique global, et la méthode agent, basée sur des modèles visant un réalisme individuel local. Mais cette création de plusieurs milliers de simulations nécessite de développer une méthode de validation automatique du réalisme local des simulations grande échelle. À la suite de Darty, Saunier, \& Sabouret (2014) nous pensons que l'obtention de classifications mixtes, c'est-à-dire comportant des emplois du temps simulés et des emplois du temps réels ${ }^{4}$, signifierait que les activités simulées ne peuvent pas être distinguées des réelles (pour les variables considérées). Ce résultat irait alors dans le sens d'une validation du réalisme local de nos simulations grandes échelles.

51 L'élargissement de la simulation à une population (un pays, une ville) nécessite de préciser le statut épistémologique de ce passage à l'échelle. La simulation grande échelle telle qu'elle est développée ne peut pas être assimilée à un niveau social supérieur d'organisation de l'activité. En créant des milliers de simulations nous créons une multitude de foyers individuels qui n'ont pas d'interaction les uns avec les autres. Cette simplification de la simulation grande échelle s'appuie sur le fait que les consommations énergétiques sont dépendantes de la dynamique interne à l'habitat et sur le fait que les emplois du temps de l'INSEE sont en partie influencés par l'environnement social des personnes enquêtées. La codétermination d'un niveau local de l'activité avec un niveau social de l'activité n'est aujourd'hui pas nécessaire dans SMACH mais elle pourrait le devenir dès lors que l'on souhaiterait faire la relation entre une évolution sociétale (par exemple, la part grandissante du développement durable dans la société) et son impact sur les pratiques dans l'habitat. Dans cette perspective il serait alors possible de donner une autre dimension au concept de modes de vie (Thomas, 2011 et 2014 ; Ravalet, \& al., 2015) actuellement intégré dans le modèle SMACH (voir la figure 3). Il pourrait à l'avenir jouer un rôle d'interface, de codétermination, entre la dimension locale de l'activité et sa dimension sociale qui serait à modéliser. La précision de ce statut épistémologique renvoie au défi de l'analyse multi-niveau telle qu'abordée par d'autres chercheurs (e.g. Roth 2001, Theureau 2015, Poizat, Durand, \& Theureau 2016). Elle pourrait donc certainement bénéficier des réflexions actuellement menées ou y contribuer, mais ce travail reste à mener.

52 Enfin, et plus largement, l'inscription de notre problématique dans le champ de l'efficience énergétique et du développement durable renvoie à la compréhension/ analyse d'un système complexe dans lequel s'entremêlent les dimensions cognitives, sociétales, techniques (bâtiments, équipements) mais aussi politiques (réglementations nationale, internationale). Actuellement la complexité des questions liées à l'efficience énergétique ouvre très souvent sur des expérimentations en situation réelle pour mettre en synergie ces différentes dimensions (activité, sociétal, politique). Ces living-lab rencontrent pourtant aussi des difficultés de réalisme, de possibles techniques et humains, de généralisation. Une nouvelle voie semble fructueuse et porte sur l'articulation entre une problématique de living-lab (expérimentation in vivo) et une problématique de monde artificiel (expérimentation in silico). Cette articulation peut alors être envisagée pour la construction et le partage des données empiriques, pour la généralisation de résultats de l'analyse empirique, pour la génération d'hypothèses à partir de l'expérimentation in silico, pour des tests, etc. L'objectif ici serait la construction d'expérimentation in vivo et in silico c'est-à-dire le développement d'un environnement 
global suffisamment réaliste pour donner la possibilité à des acteurs industriels, politiques et particuliers de décider en articulant les trois dimensions: technique, cognitive et sociétale.

\section{BIBLIOGRAPHIE}

Béguin, P., \& Weill-Fassina, A. (1997). La simulation en ergonomie : connaître, agir et interagir. Octarès. Toulouse.

Bellamine-Ben Saoud, N., Ben Mena, T., Dugdale, J., Pavard, B., \& Ben Ahmed, M. (2006). Assessing large scale emergency rescue plans : an agent-based approach. The International Journal of Intelligent Control and Systems, 11, 260-271.

Darty, J., Saunier, J., \& Sabouret, N. (2014). Behavior Clustering and Explicitation for the Study of Agents' Credibility : Application to a Virtual Driver Simulation. In Conf. Agents Artif. Intell., pp. 8299.

Delenne, B. (2017). Analyses des 1000 simulations SMACH. Document interne EDF R\&D Mémo E74/17/012/A.

Dugdale, J., Pavard, B., \& Soubie, J. L. (2000) A pragmatic development of a computer simulation of an emergency call centre. In Dieng, R. Giboin, A., Karsenty, L., \& De Michelis, G. (Eds.), Designing Cooperative Systems - Proceedings of the 4th International Conference on the Design of Cooperative Systems (pp. 241-256). Amsterdam : IOS Press.

Fanger, P. O., (1970). Thermal confort : Analysis and applications in environmental engineering. Danish Technical Press.

Ferber, J. (1995). Les systèmes Multi-agents : vers une intelligence collective. InterÉditions.

Gallagher, S. A., Reinerman-Jones, L., Sollins, B., \& Janz, B. (2014). Using a simulated environment to investigate experiences reported during space travel. Theoretical Issues in Ergonomics Science, vol. 15 , no. 4, pp. 376-394.

Gargiulo, F., Ternes, S., Huet, S., \& Deffuant, G. (2010). An Iterative Approach for Generating Statistically Realistic Populations of Households. PLoS ONE 5(1) Editor : Fabio Rapallo, University of East Piedmont, Italy.

Guibourdenche, J. (2013). Préoccupations et agencements dans les contextes d'activité domestique : contribution à la conception de situations informatiques diffuses, appropriables et énergétiquement efficaces. Thèse de doctorat Lyon 2.

Guibourdenche, J., Vacherand-Revel, J., Fréjus M., \& Haradji Y. (2015). Analyse de contextes d'activité domestique pour la conception de systèmes diffus énergétiquement efficients. Activités 12-1, mis en ligne le 15 avril 2015. URL : http://activites.revues.org/994 ; DOI : 10.4000/ activites.994.

Grandjean, A. (2013). Introduction de non linéarités et stationnarités dans les modèles de représentation de la demande électrique résidentielle. Thèse de doctorat en énergétique. Mines ParisTech, Paris. 
Haradji, Y., Poizat, G., \& Sempé, F. (2012). Human Activity and social simulation. In Advances in applied human modeling and simulation, p. 416-425. CRC Press.

Haradji, Y., \& Poizat, G., (2017). Simuler l'activité humaine. Dans Encyclopédie d'analyse des activités . Sous la direction de Barbier, JM. \& Durand, M. p. 255-280. PUF.

Haradji, Y., \& Sempé, F., (2015). Valider la simulation de comportements humains dans SMACH : principes méthodologiques et résultats. EDF R\&D. Note H-E76-2015-05747.

Haué, J.-B. (2004). Intégrer les aspects situés de l'activité dans une ingénierie cognitive centrée sur la situation d'utilisation. Activités, 1(2), 170-194.

Huraux, T. (2015). Simulation multi-agent d'un système complexe : combiner des domaines d'expertise par une approche multi-niveau - Le cas de la consommation électrique résidentielle. Thèse de doctorat en informatique. Université Pierre et Marie Curie, Paris.

Huraux, T., Sabouret, N., \& Haradji, Y. (2014). A multi-level Model for Multi-Agent Based Simulation. In Proc of the 6th International Conference on Agents and Artificial Intelligence (ICAART). Angers, France.

Kashif, A. (2014). Modélisation du comportement humain réactif et délibératif avec une approche multiagent pour la gestion énergétique dans le bâtiment. Thèse de doctorat, Université de Grenoble.

Lefèvre, A. (2016). Simulation Sociale et simulacre structural. Variations [En ligne], 19/2016. Mis en ligne le 06/04/2016. URL : http://variations.revues.org/720.

Lee Y, S., Yi., \& Malkawi, A. (2011). Simulating Human Behavior and its Impact on Energy Uses. In Proc. Of the 12th Conference of International Building Performance Simulation Association (IBPSA), pages 1049-1056.

Manzo, G. (2007). Potentialités et limites de la simulation multi-agents : une introduction. Revue française de sociologie, 55, 653-688.

Maturana, H., \& Varela, F. (1994 version française). L'arbre de la connaissance. Racines biologiques de la compréhension humaine. Editions Addison-Wesley France, S.A.

Pavard, B., \& Dugdale, J. (2006). The contribution of complexity theory to the study of sociotechnical cooperative systems. In A. Minai \& Y. Bar-Yam (Eds.), Unifying Themes in Complex Systems - Proceedings from the $3^{\text {rd }}$ International Conference on Complex Systems (pp. 39-48). Heidelberg, Springer.

Phan, D., \& Amblard, F., (2007). Multi-Agent Modeling and Simulation in the social and Human Sciences. Barwell Press, GEMAS Studies. In Social Analysis.

Phan, D., \& Varenne, F. (2010). Agent-Based Models and Simulations in Economics and Social Sciences : From Conceptual Exploration to Distinct Ways of Experimenting. Journal of Artificial Societies and Social Simulation. 13 (1) 5. http://jasss.soc.surrey.ac.uk/13/1/5.html.

Plessis, G., Amouroux, E., \& Haradji, Y. (2014). Coupling occupant behavior with a building energy model - A FMI application. The 10th International Modelica Conference.

Poizat, G., Durand, M., \& Theureau, J. (2016). The challenges of activity analysis for training objectives. Le Travail Humain, 79(3), 233-258.

Poizat, G., Fréjus, M., \& Haradji, Y. (2009). Analysis of activity in domestic settings for the design ubiquitous technologies. In Norros, L., Koskinen, H., Salo, L. \& Savioja, P. (Eds.), Proceedings of the European Conference on Cognitive Ergonomics : Designing beyond the Product - Understanding Activity and User Experience in Ubiquitous Environments (pp. 145-146). Helsinki, VTT. 
Ravalet, E., Tabbone, L., Kaufmann, V., Durand-Daubin, M., \& Cayla, JM. (2015). Modélisation du rôle des modes de vie dans les consommations d'énergie. Ecole polytechnique Fédérale de Lausanne, Report-220215.

Reynaud, Q., Sempé, F., Haradji, Y., \& Sabouret, N. (2017). Simuler l'activité humaine en combinant un système multi-agent avec des approches statistiques basées sur les "enquêtes emploi du temps”. Journées Francophones sur les Systèmes Multi-Agents (JFSMA 2017), July 2017, Caen, France.

Roth, W.-M. (2001). Situating cognition. The Journal of the Learning Sciences, 10(1/2), 27-61.

Salembier, P., Dugdale, J., Fréjus, M., \& Haradji, Y. (2009). A descriptive Model of contextual activities for the design of domestic situation. Proceeding European Conference on Cognitive Ergonomics : Designing beyond the Product. Otaniemi, Finland.

Salembier, P., \& Pavard, B. (2004). Analyse et modélisation des activités coopératives situées. Evolution d'un questionnement et apports à la conception. Activités, 1, 87-99.

Sanders, L. (2007). Models in Spatial Analysis. London : ISTE.

Theureau J. (2004) Le cours d'action : méthode élémentaire. Octarès, Toulouse.

Theureau, J. (2015). Le cours d'action. L'énaction et l'expérience. Octarès, Toulouse.

Thomas, M.-P. (2011) « Energy consumption in residential and transport sectors : an analysis based on a lifestyle approach ». In 4th ECLEER Seminar (European Centre and Laboratories for Energy E ficiency Research), EDF R\&D.

Thomas, M.-P. (2014). Les choix résidentiels : une approche par les modes de vie, in Mobilités résidentielles, territoires et politiques publiques. Le regard sociologique coll.

Van Belleghem, L., \& Guerry, M-H. (2016). De la simulation organisationnelle à la simulation managériale, Actes du $51^{\text {ème }}$ Congrès de la SELF, Marseille, 21-23/09/16

Varela, F. (1989). Autonomie et connaissance. Paris, Seuil.

Winograd, T., \& Flores, F. (1989). L'Intelligence Artificielle en question. Paris, PUF.

\section{NOTES}

1. Ces travaux ont été réalisés, pour la partie informatique, en collaboration entre EDF, l'UPMC (Université Pierre et Marie Curie), l'IRD (Institut de Recherche pour le Développement) et le LIMSI (Laboratoire de recherche en Informatique du CNRS).

2. Nous disposons ainsi de carnets qui correspondent aux emplois du temps précis et diversifiés de 27000 personnes. Nous reconstruisons alors, pour chaque profil issu de la génération de population, les actions régulières, celles irrégulières et leur séquencement.

3. Comme indiqué précédemment nous ne présentons pas dans ce texte les résultats relatifs à la validation quantitative des consommations.

4. Les emplois du temps réels sont créés à partir de l'enquête emploi du temps de l'INSEE (voir section 2.2) et comparés aux emplois du temps tels qu'ils sont mis en œuvre dans la simulation. 


\section{RÉSUMÉS}

SMACH (Simulation Multi-Agent de l'ACtivité Humaine) est une plateforme de simulation sociale qui s'inscrit pour EDF dans un cadre de recherche visant à utiliser la simulation pour l'anticipation et la réduction des consommations d'énergie dans l'habitat. Dans cet article, nous développons l'idée que la simulation nécessite d'intégrer une relation structurelle à l'activité humaine, car la consommation énergétique dans l'habitat résulte pour beaucoup de la dynamique de vie quotidienne. Pour cela, nous concevons le réalisme de la simulation en articulant deux niveaux de modélisation de l'activité humaine. Le premier niveau a consisté à cadrer la conception avec trois hypothèses épistémologiques de l'énaction et du cours d'action (activité individuelle, collective et couplage avec l'environnement). Le deuxième niveau de modélisation a consisté à décliner ces hypothèses sur la base de notre connaissance empirique de l'activité dans l'habitat. Nous explicitons, pour chacune de ces hypothèses, des proximités et des différences entre les connaissances issues de l'analyse empirique et les dynamiques créées dans la simulation. La plateforme SMACH est aujourd'hui sollicitée par des experts métiers qui s'appuient sur les diagrammes d'activité humaine ou sur les courbes de consommation d'énergie pour réaliser des études relatives à l'utilisation future de l'électricité.

SMACH (Multi-Agent Human Activity Simulation) is a social simulation platform that is part of EDF's Research and Development framework aimed at developing simulation to anticipate and reduce energy consumption in homes. In this paper, we develop the idea that simulation requires the integration of a structural relationship into human activity, as residential energy consumption is largely the result of daily life dynamics. To this end, we show that the realism of the simulation realism is created by combining two levels of human activity modeling. The first level consists in framing the design with three epistemological hypotheses of the enaction and the Course of Action (individual, collective activity and coupling with the environment). The second level of modeling consists in applying these hypotheses based on our empirical knowledge of activity in real households. For each of these hypotheses, we explain the proximity and differences between the knowledge derived from empirical analysis and the dynamics created in the simulation. The SMACH platform is currently used by business experts who rely on human activity diagrams or energy consumption curves to carry out studies on the future use of electricity.

\section{INDEX}

Keywords : multi-agent simulation, activity modeling, enaction, course of action, consumption curves, energy efficiency

Mots-clés : simulation, multi-agent, modélisation de l'activité, énaction, cours d'action, courbes de consommation, efficience énergétique 


\section{AUTEURS}

\section{YVON HARADJI}

EDF R\&D

yvon.haradji@edf.fr

\section{JULIEN GUIBOURDENCHE}

ERSYA

jguibourdenche@ersya.com

QUENTIN REYNAUD

QRCI

quentin.reynaud.pro@gmail.com

\section{GERMAIN POIZAT}

Université de Genève, Faculté de Psychologie et des Sciences de l'Education Germain.Poizat@unige.ch

\section{NICOLAS SABOURET}

LIMSI - CNRS - UPR 3251 - Université Paris-Sud

nicolas.sabouret@limsi.fr

\section{FRANÇOIS SEMPÉ}

FSCI

sempe.francois@gmail.com

THOMAS HURAUX

SCALIAN, Bordeaux

thomas.huraux@scalian.com

MARIANE GALBAT

ERSYA

mgalbat@ersya.com 